\title{
Crystallization of Poly(ethylene oxide) from Solutions of Different Solvents
}

\author{
Takashi SASAKI, ${ }^{\dagger}$ Akane MiYAzAKI, Shuma SugIURA, and Koji OKADA \\ Department of Materials Science \& Engineering, Fukui University, Fukui 910-8507, Japan
}

(Received March 28, 2002; Accepted September 13, 2002)

\begin{abstract}
The kinetics and crystalline morphology for isothermal crystallization of poly(ethylene oxide) (PEO) from solutions in three different solvents, $N, N$-dimethylacetamide (DMAc), toluene, and tripropionin (TP), were investigated. The energy barrier $A^{\prime}$ of primary nucleation was highest for PEO/TP, and that of radial growth $C^{\prime}$ was highest for PEO/toluene. Crystallites grew from both the DMAc and toluene solutions as aggregates of small layers of lamellae with branching and splitting. In addition, the crystallites from PEO/DMAc were found to have polygonal-like contours outside the lamella aggregates. This finding suggests that liquid-liquid phase separation occurred before or during the crystallization for PEO/DMAc. Relatively large spherulites were obtained from PEO/TP solutions due to the relatively low primary nucleation rate. The number density of the crystallites from PEO/TP was much lower than those from $\mathrm{PEO} / \mathrm{DMAc}$ and $\mathrm{PEO} /$ toluene.

KEY WORDS Poly(ethylene oxide)/ Crystallization from Solutions / Primary Nucleation / Radial Growth / Solvent Effect / Morphology /
\end{abstract}

Since polymer crystallization is essentially a kinetically-controlled process, the morphology of polymer crystals grown from solutions is very different from that in the case of bulk or melt crystallization due to the effects of polymer-solvent interaction, solution viscosity, concentration, molecular weight, and various other factors. The effect of solution concentration on the rate of crystallization can be treated by introducing an excess entropy term into the kinetic nucleation theory. ${ }^{1-3}$ Nakajima et al. investigated the solvent effect on single crystal formation of polyethylene from various solutions, and they revealed that the thermodynamic interaction with solvent plays a dominant role. ${ }^{4,5}$ However, studies on the solvent effect have been done exclusively for single crystals from very dilute solutions, and the general characteristics of the solvent effect on solution crystallization have not yet been fully elucidated even from the phenomenological point of view. In some cases, strong interaction between a polymer and a solvent results in the formation of a polymersolvent compound ${ }^{6,7}$ or in physical gelation, ${ }^{8-11}$ while in other cases, a liquid-liquid phase separation process may play a role in the crystallization. ${ }^{10,12,13}$ However, the effects of liquid-liquid phase separation on kinetics and crystalline morphology have not been extensively studied.

In our recent research on crystallization of poly(ethylene oxide) (PEO) from non-dilute solutions of a very viscous solvent, tripropionin (TP), we have found that the heterogeneity arising from incomplete dissolution in the initial solution plays an important role in the primary nucleation. ${ }^{14,15}$ This is related to the so-called

${ }^{\dagger}$ To whom correspondence should be addressed. melt memory effect reported for melt crystallization. ${ }^{17}$ The significance of this phenomenon should be renewedly assessed in the light of recent issue of the crystallization mechanism at very early stages. ${ }^{16,18} \mathrm{We}$ have also revealed a unique behavior specific to the $\mathrm{PEO} / \mathrm{TP}$ system; that is, large spherulites of up to several $\mathrm{mm}$ in diameter grow at a very low rate of the primary nucleation, ${ }^{14}$ without any sign of liquid-liquid phase separation during crystallization or of formation of a polymer-solvent compound.

Motivated by our awareness of the importance of the solvent effect, we have been exploring various solvents for the crystallization of PEO. In this study, we examined the kinetics and morphology of isothermal crystallization of PEO in three different solvents, $\mathrm{N}, \mathrm{N}$ dimethylacetamide (DMAc), toluene, and TP, by a direct observation technique using a CCD camera and by optical microscopy.

\section{EXPERIMENTAL}

Commercial PEO $\left(M_{\mathrm{n}}=2.04 \times 10^{-4}, M_{\mathrm{w}}=2.16 \times\right.$ $10^{-4}$ ) was used without further purification. DMAc, toluene, and TP were distilled under reduced pressure and were filtered using a $0.2-\mu \mathrm{m}$ mesh Millipore filter just before use. PEO was added to these solvents in 1 mm-thick quartz cells to make solutions of lower ( 0.5 and $1.0 \mathrm{wt} \%$ PEO content) and higher concentrations $(30 \mathrm{wt} \%)$ in a dust-free atmosphere, and they were degassed and sealed off under vacuum $\left(<10^{-5} \mathrm{mmHg}\right)$. These samples were further pre-annealed for $200 \mathrm{~h}$ at $120^{\circ} \mathrm{C}$ for PEO/DMAc, $90^{\circ} \mathrm{C}$ for $\mathrm{PEO} /$ toluene, and $200^{\circ} \mathrm{C}$ for PEO/TP before the kinetics measurements of crystallization. We have already shown in a previ- 
Table I. Equilibrium parameters for $30 \mathrm{wt} \%$ solutions and for solvents

\begin{tabular}{lccc}
\hline & DMAc & Toluene & TP \\
\hline$T_{\mathrm{d}}{ }^{\circ}(\mathrm{K})$ & 314 & 313 & 322 \\
$\Delta H_{\mathrm{d}}\left(10^{-9} \mathrm{erg} \mathrm{cm}^{-3}\right)^{\mathrm{a}}$ & 3.23 & 2.04 & 2.29 \\
$\Delta S_{\mathrm{d}}\left(10^{-6} \mathrm{erg} \mathrm{K}^{-1} \mathrm{~cm}^{-3}\right)^{\mathrm{b}}$ & 10.3 & 6.52 & 7.11 \\
$\eta$ at $20^{\circ} \mathrm{C}(\mathrm{cP})^{\mathrm{c}}$ & 0.92 & 0.58 & 6.08 \\
$E_{\eta}\left(\mathrm{kcal} \mathrm{mol}^{-1}\right)^{\mathrm{d}}$ & 1.80 & 1.26 & 6.5 \\
\hline
\end{tabular}

${ }^{\mathrm{a}}$ Enthalpy of dissolution per unit volume of the bulk crystal of PEO. ${ }^{b}$ Entropy of dissolution per unit volume of the bulk crystal of PEO. ${ }^{c}$ Viscosity of solvent. ${ }^{\mathrm{d}}$ Activation energy of the solvent viscosity.

ous study that the crystallization rate decreases with increase in the pre-annealing time for a PEO/TP system. ${ }^{14,15}$ The primary nucleation rate $I$ and the final asymptotic value of the number density of crystallites $n_{\mathrm{f}}$ showed remarkable decreases with increase in preannealing time. These findings suggested heterogeneous nucleation due to incomplete dissolution on a microscopic order. The same phenomena were observed for all of the solutions used in the present study. We have confirmed that pre-annealing under the above condition gives a constant value of $I$ for $30 \mathrm{wt} \%$ solutions regardless of pre-annealing time.

In each run of the isothermal crystallization experiment, the sample mixture was again annealed for $8-10 \mathrm{~min}$ at the pre-annealing temperature mentioned above and then quenched to the crystallization temperature. The rates of primary nucleation $I$ and radial growth $G$ were measured by examining changes in the number density $n(t)$ and diameter $d(t)$ of crystallites with crystallization time from images taken by a CCD camera recorded by a videotape recorder. Here, $d(t)$ was estimated as the length of a specific side of a specific crystallite for each run of the isothermal crystallization experiment. The procedure used for measurements was described in detail in our previous paper. ${ }^{15}$ The equilibrium dissolution temperature $T_{\mathrm{d}}{ }^{\circ}$ and enthalpy of dissolution $\Delta H_{\mathrm{d}}$ for each solvent were determined in the same manner as described previously. ${ }^{15}$ The obtained values are listed in Table I. The morphology of the obtained crystallites was observed using a polarizing optical microscope (Olympus BHSP-751).

\section{RESULTS AND DISCUSSION}

\section{Primary Nucleation}

The number density of crystallites $n(t)$ for the samples of $30 \mathrm{wt} \%$ PEO content gave a nonlinear profile in which the slope decreases with respect to crystallization time, suggesting a heterogeneous nucleation. This may be partially due to the micro-residuals of incomplete dissolution that remain in the solution in spite of

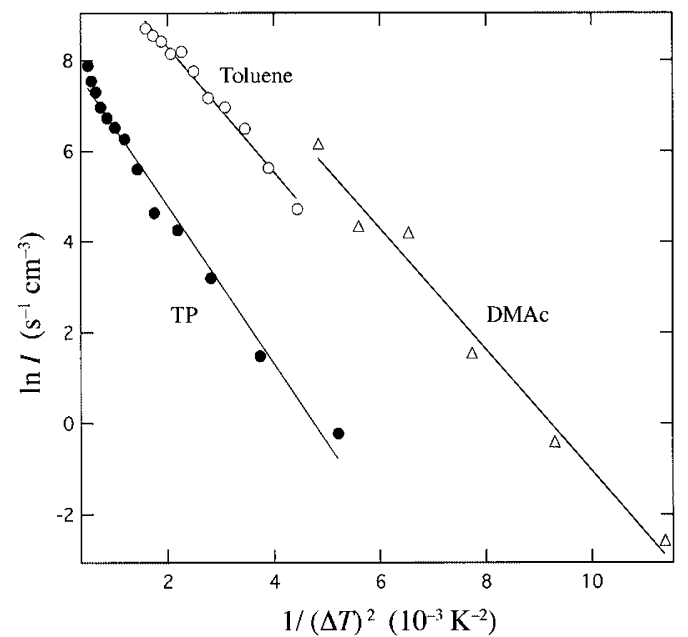

Figure 1. Plots of $\ln I$ against $\Delta T^{-2}$ for $30 \mathrm{wt} \%$ solutions of DMAc, toluene, and TP. The solid lines indicate results of the leastsquares fitting analysis.

the sufficient pre-annealing to achieve constant $I$ with respect to pre-annealing time. ${ }^{15}$ Very small impurities (smaller than $0.2 \mu \mathrm{m}$ ) may also act as seeds of nucleation. The obtained $n(t)$ curves were fitted to the following empirical equation: ${ }^{15}$

$$
n(t)=n_{\mathrm{f}}\left\{1-\exp \left[-\alpha\left(t-t_{0}\right)\right]\right\}
$$

where $t_{0}$ is the induction period. The primary nucleation rate was estimated as $I=n_{\mathrm{f}} \alpha$, which means the initial slope of the curve. Taking into account the contribution of entropy of polymer separation from a solution, ${ }^{1,2}$ the kinetic nucleation theory ${ }^{19,20}$ leads to a concentration-dependent expression, ${ }^{15}$

$$
I=I_{0} \exp \left[-A^{\prime} /(\Delta T)^{2}\right]
$$

with

$$
A^{\prime}=A_{1}-A_{2} \ln c
$$

where $A_{1}$ and $A_{2}$ have little temperature dependence (almost constant) compared with $(\Delta T)^{-2}$ in the temperature range of the present study, and $c$ is the volume fraction of the polymer of the initial solution. Note that in eq 2, a diffusion term is included in the preexponential factor $I_{0}$. This expression can be used when the temperature dependence of the diffusion term is negligibly weak compared with that of the entropy term. Figure 1 shows examples of $\ln I$ plotted against $(\Delta T)^{-2}$ for $30 \mathrm{wt} \%$ solutions. These data exhibit a good linear relationship, indicating that eq 2 is applicable to the primary nucleation process of our systems. Table II shows values of $I$ at a typical supercooling, $\Delta T=15 \mathrm{~K}$, revealing that the primary nucleation occurs fastest for $\mathrm{PEO} / \mathrm{DMAc}$ and slowest for PEO/TP. This may be partially due to a high viscosity of TP: the PEO chains have to travel through the solvent molecules (translational 
Table II. Absolute values of $I$ and $G$ for $30 \mathrm{wt} \%$ solutions at $\Delta T=15 \mathrm{~K}$

\begin{tabular}{lccc}
\hline & DMAc & Toluene & TP \\
\hline$I\left(\mathrm{~cm}^{-3} \mathrm{~s}^{-1}\right)$ & 473 & 111 & 1.53 \\
$G\left(10^{-3} \mathrm{~mm} \mathrm{~s}^{-1}\right)$ & 0.721 & 1.62 & 6.70 \\
\hline
\end{tabular}

Table III. Parameters obtained from the rate measurements for $30 \mathrm{wt} \%$ solutions

\begin{tabular}{lccl}
\hline & DMAc & Toluene & TP \\
\hline$c$ & 0.246 & 0.232 & 0.274 \\
$A^{\prime}\left(10^{3} \mathrm{~K}^{2}\right)$ & 1.33 & 1.38 & 1.85 \\
$C^{\prime}\left(10^{3} \mathrm{~K}\right)$ & 0.192 & 0.242 & 0.0624 \\
$E_{\mathrm{a}}{ }^{\prime}\left(\mathrm{kcal} \mathrm{mol}^{-1}\right)$ & 108 & 63.6 & 4.74 \\
\hline
\end{tabular}

diffusion) to form a primary nucleus, and the higher viscosity of TP may therefore reduce the absolute values of $I$.

Table III shows the values of $A^{\prime}$ obtained from the slope of $\ln I$ against $(\Delta T)^{-2}$ for $30 \mathrm{wt} \%$ solutions, which is a measure of the energy barrier for the primary nucleation. It can be seen that $A^{\prime}(\mathrm{TP})>A^{\prime}$ (toluene $) \approx$ $A^{\prime}$ (DMAc). For the PEO/DMAc and PEO/TP solutions of lower concentrations ( 0.5 and $1.0 \mathrm{wt} \%), I$ could not be estimated with sufficient accuracy by the present method: a tremendous number of crystallites appeared and were rapidly precipitated in the former solution, and the number of crystallites in the latter solution was too small to achieve sufficient accuracy.

\section{Radial Growth}

In the PEO/DMAc and PEO/toluene systems, we found that the diameter $d(t)$ of crystallites increases linearly in the early stage of crystallization but that the radial growth rate later decreases with time, especially in solutions of lower concentrations ( 0.5 and $1.0 \mathrm{wt} \%)$. Observation by the CCD camera showed that there was little collision between the crystallites within the measurement period, suggesting that the decrease in radial growth rate is mainly due to the decrease in PEO content in the solution phase as the crystallization proceeds. On the other hand, little decrease in the radial growth rate was observed in the PEO/TP system, and linear growth continued until the spherulites began to collide with each other. The overall crystallization rate for $\mathrm{PEO} / \mathrm{TP}$ was much lower than those for PEO/DMAc and $\mathrm{PEO} /$ toluene, but huge crystallites grew from $\mathrm{PEO} / \mathrm{TP}$ (typically several $\mathrm{mm}$ in final diameter) until the entire space in the sample cell was filled with crystallites. The crystallites grown from DMAc and toluene solutions were much smaller: the final diameters of crystallites grown from DMAc and toluene solutions were typically 0.2 and $1.0 \mathrm{~mm}$, respectively, at $\Delta T=20 \mathrm{~K}$ for $30 \mathrm{wt} \%$ solutions. The number densities of crystallites of $\mathrm{PEO} / \mathrm{DMAc}$ and $\mathrm{PEO} /$ toluene were

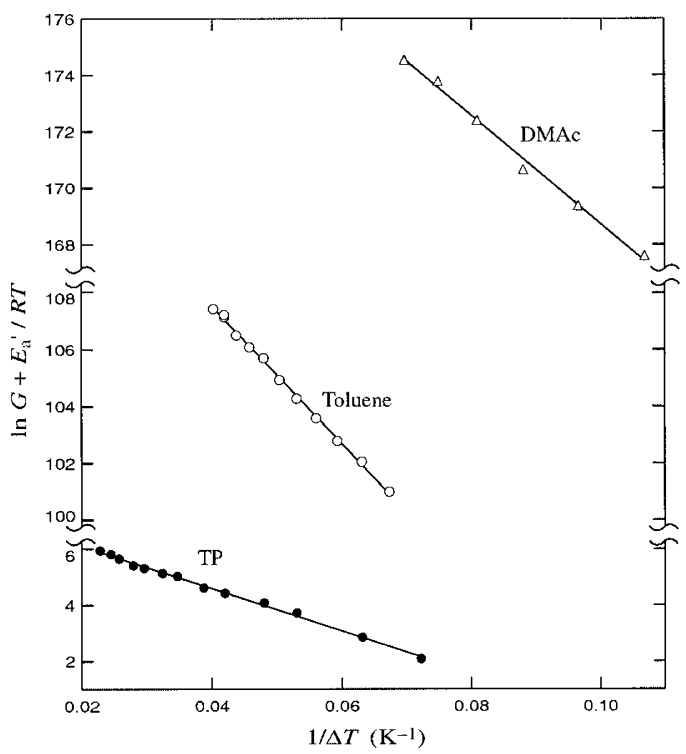

Figure 2. Plots of $\ln G+E_{\mathrm{a}}{ }^{\prime} / R T$ against $\Delta T^{-1}$ for $30 \mathrm{wt} \%$ solutions of DMAc, toluene, and TP.

much higher than that of PEO/TP. For PEO/DMAc, the number density was often too large to measure by our technique. The results in Table II showing that $I / G(\mathrm{DMAc})>I / G$ (toluene) $>I / G(\mathrm{TP})$ are consistent with this observation.

The radial growth rate $G$ was obtained from the initial slope of the diameter vs. time data. Again, by introducing the entropy contribution of polymer separation from a solution to the surface nucleation theory, ${ }^{20,21}$ $G$ is expressed as ${ }^{1,2}$

$$
G=G_{0} \exp \left(-C^{\prime} / \Delta T\right) \exp \left(-E_{\mathrm{a}}{ }^{\prime} / R T\right)
$$

with

$$
C^{\prime}=C_{1}-C_{2} \ln c
$$

where $E_{\mathrm{a}}{ }^{\prime}$ is the apparent activation energy, $R$ is the gas constant, and parameters $C_{1}$ and $C_{2}$ have little temperature dependence compared with $(\Delta T)^{-1}$ in the temperature range used in the present study. Figure 2 shows typical profiles of $\ln G+E_{\mathrm{a}}{ }^{\prime} / R T$ for $30 \mathrm{wt} \%$ solutions. We analyzed $G$ vs. $T$ data by the nonlinear least-squares fitting method to obtain the best fit set of $C^{\prime}$ and $E_{\mathrm{a}}{ }^{\prime}$. The results are shown in Table III. From Tables II and III, it can be seen that $C^{\prime}$ (toluene) $>C^{\prime}($ DMAc $)>C^{\prime}(\mathrm{TP})$ and also that the absolute values of $G$ are smaller for PEO/DMAc and $\mathrm{PEO} / \mathrm{DMAc}$ than for $\mathrm{PEO} / \mathrm{TP}$. We should remember the finding of relatively high primary nucleation rates for PEO/DMAc and PEO/toluene. It is likely that a larger amount of PEO is incorporated in the primary nucleation event, resulting in a greater reduction in the PEO content of the solution phase when the crystallites have grown to sizes that are detectable by the CCD camera ( $c a$. a few tens of $\mu \mathrm{m}$ in diameter). Therefore, our data of $G$ for $\mathrm{PEO} / \mathrm{DMAc}$ and $\mathrm{PEO} /$ toluene 
seem to reflect the radial growth from a solution of less PEO content than in the case of PEO/TP. Considering the concentration dependence of $C^{\prime}$ in eq 5 , we see that higher $I$ is responsible for higher $C^{\prime}$ for PEO/DMAc and PEO/toluene. Similarly, the lower $G$ for $\mathrm{PEO} / \mathrm{DMAc}$ and $\mathrm{PEO} /$ toluene can be accounted for by the above consideration of the lower PEO content of the solution phase.

Table III also shows that the apparent activation energy $E_{\mathrm{a}}{ }^{\prime}$ of $\mathrm{PEO} / \mathrm{DMAc}$ and that of $\mathrm{PEO} /$ toluene are much greater than that of PEO/TP. This extreme difference suggests an essential difference in the diffusion mechanisms during the process of radial growth. In DMAc and toluene solutions, larger-scaled motions of the PEO chain might be required for polymer deposition onto the growth front (or surface nucleation) than that in TP. As stated above, the PEO content of the solution phase from which radial growth occurs seems to be lower for DMAc and toluene than for TP, and in turn, this might be responsible for the difference in the diffusion mechanisms. Furthermore, for the PEO/DMAc system, liquid-liquid phase separation might influence the crystallization process as is presented in the following section. Thus, the apparent value of $E_{\mathrm{a}}{ }^{\prime}$ for this system may reflect a rather complicated diffusion process including the phase separation. We also note that the activation energy of viscosity $E_{\eta}$ exceeds $E_{\mathrm{a}}{ }^{\prime}$ for the $\mathrm{PEO} / \mathrm{TP}$ system. This suggests a weak dynamical interaction between PEO and TP, i.e., Kramers low-friction limit $^{22}$ seems to hold for this system as we have discussed in a previous paper. ${ }^{15}$ This may be specific to the solvent TP; we have found a similar behavior for an isotactic polystyrene/TP system. ${ }^{23}$

\section{Morphology}

It is known that PEO forms single crystals from a very dilute solution (e.g., $0.01 \mathrm{wt} \%$ PEO content). ${ }^{24,25}$ Morphological studies have been extensively done for single crystals of PEO (PEO-polystyrene copolymers) formed under various conditions from dilute solutions. $^{26,27}$ In contrast, we found that the concentrations used in the present study $(0.5,1.0$, and $30 \mathrm{wt} \%)$ are not low enough to obtain single crystals. It has been revealed that for crystallization of polyethylene from solutions, lamella thickness as well as growth rate depends strongly on rather the degree of supercooling than the polymer concentration. ${ }^{28-30}$ Based on this finding for polyethylene which suggests a less prominent effect of solvent on crystallization behavior, our results on morphology presented below may be surprising: crystalline morphology in our systems dramatically changes with solvent. Thus, the solvent effect on morphology would be an interesting issue.
Figures 3, 4, and 5 show optical micrographs of the crystallites formed by isothermal crystallization in DMAc, toluene, and TP solutions of $1.0 \mathrm{wt} \%$, respectively. These micrographs were taken in situ in the presence of solution phase after a long crystallization time, when the crystallization had almost ceased. In

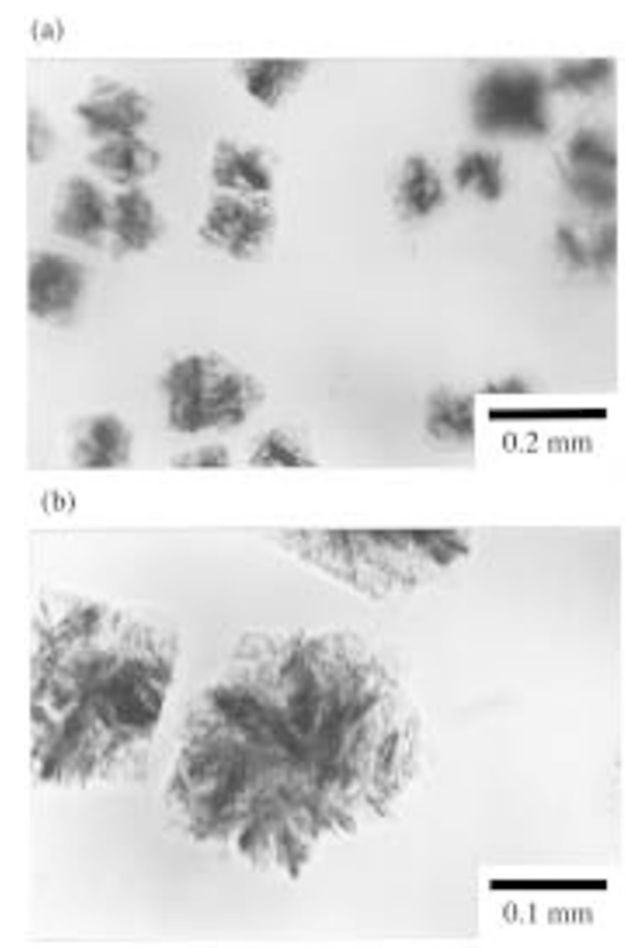

Figure 3. Optical micrographs of PEO crystallites grown from $1.0 \mathrm{wt} \%$ DMAc solution at (a) $\Delta T=13 \mathrm{~K}$ and (b) $\Delta T=23 \mathrm{~K}$.

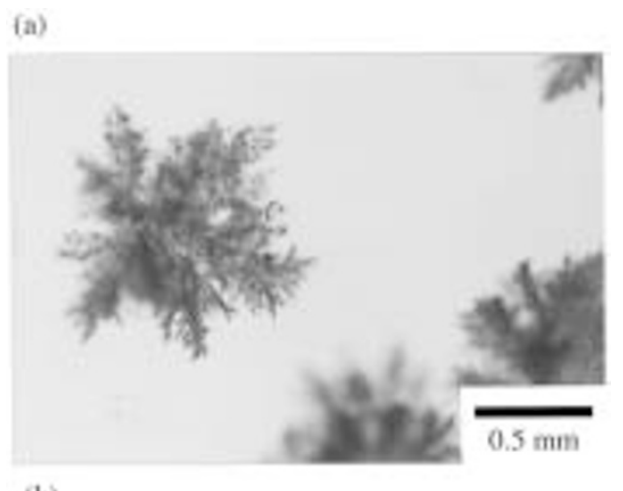

(b)

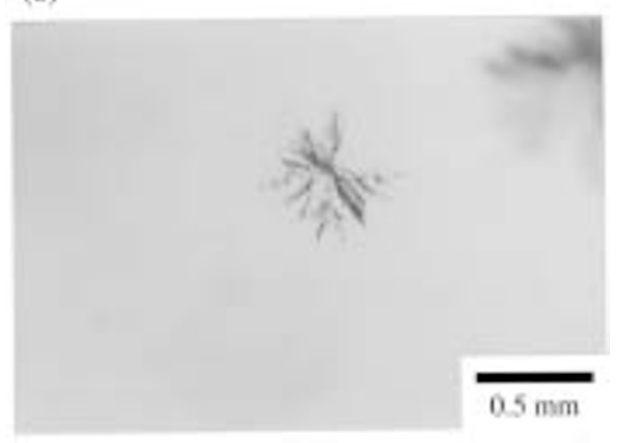

Figure 4. Optical micrographs of PEO crystallites grown from $1.0 \mathrm{wt} \%$ toluene solution at (a) $\Delta T=13 \mathrm{~K}$ and (b) $\Delta T=23 \mathrm{~K}$. 

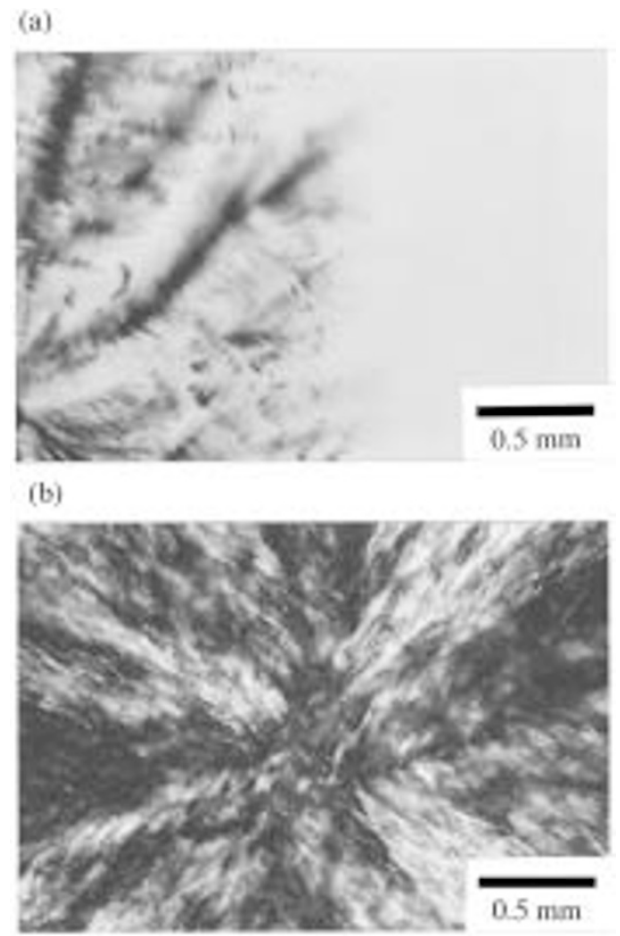

Figure 5. Optical micrographs of PEO crystallites grown from $1.0 \mathrm{wt} \% \mathrm{TP}$ solution at (a) $\Delta T=17 \mathrm{~K}$ and (b) $\Delta T=27 \mathrm{~K}$.

PEO/DMAc solutions (Figure 3), we can see that a number of small layers or rods, each of which is probably composed of multi-layered lamellae or axialites, aggregate to form a crystallite. Branching and splitting of such layers are also seen. Polarizing optical microscopy revealed that the PEO chains align perpendicular to the long axis of the rod (to the surface of the lamella layer). In addition, we can see an apparent polygonal-like contour outside the ragged aggregate of lamella layers, which suggests a liquid-liquid phase separation as we will discuss later. This morphological feature was observed for 0.5 and $1.0 \mathrm{wt} \%$ solutions at $\Delta T=13-23 \mathrm{~K}$, and for $30 \mathrm{wt} \%$ solution at $\Delta T=10$ $15 \mathrm{~K}$. At $\Delta T$ of higher than $15 \mathrm{~K}$, the $30 \mathrm{wt} \%$ solution rapidly became turbid, and this change was followed by immediate precipitation of chunks; thus, we could not examine the detailed morphology.

As shown in Figure 4, the morphological features of the PEO/toluene system are similar to those of the PEO/DMAc system. However, unlike the PEO/DMAc system, we see that the layers of lamellae in the $\mathrm{PEO} /$ toluene system tend to grow with considerable branching, and also that there is no contour surrounding each crystallite. The latter finding suggests that the rate of crystallization is faster than that of the liquid-liquid phase separation or that liquid-liquid phase separation does not essentially occur under the present isothermal conditions. For PEO/toluene, the same morphological features were observed for 0.5 and $1.0 \mathrm{wt} \%$ solutions at $\Delta T=13-23 \mathrm{~K}$, and for $30 \mathrm{wt} \%$ solution at $\Delta T=15-$
$25 \mathrm{~K}$.

Crystallites from TP solutions generally grew to more than several $\mathrm{mm}$ in diameter, which is much greater than the sizes of crystallites grown from the other two solvents. We note here that there is no contour outside each of the crystallites, suggesting that the liquid-liquid phase separation does not play a role (or does not occur) in the PEO/TP system under the present conditions. Our visual observations showed that crystallites grown from PEO/TP solutions of 0.5 and $1.0 \mathrm{wt} \%$ were translucent through a $1 \mathrm{~mm}$-thick cell, thus, we infer that the solution phase (TP-rich phase) is included inside the crystallites. The morphology of crystallites from 0.5 and $1.0 \mathrm{wt} \%$ solutions varies in a range $\Delta T=17-27 \mathrm{~K}$ as shown in Figure 5. At $\Delta T=17 \mathrm{~K}$, ribbon-like bundles of lamellae, of which the thicknesses and widths are several $\mu \mathrm{m}$ and several tens of $\mu \mathrm{m}$, respectively, grow with branching to form a large crystallite. At $\Delta T=27 \mathrm{~K}$, the branching occurs more frequently than that at $\Delta T=$ $17 \mathrm{~K}$, and consequently, a large spherical crystallite (negative spherulite) is formed: it may be a common phenomenon that the rate of branching due to dislocation becomes greater as $\Delta T$ increases. ${ }^{31,32}$ Figure $5 \mathrm{~b}$ shows just a center portion of such a large spherulite. Many lamella layers (each probably composed of multi-layered lamellae) forming clusters by branching and splitting can be seen.

We now discuss implication of the contour surrounding each crystallite observed for the PEO/DMAc system (see Figure 3). We assume that an upper critical solution temperature for the PEO/DMAc system exists, below which a liquid-liquid phase separation takes place, and that there is a region where the phase separation occurs without crystallization. This type of phase diagram has been reported for polyethylene in some solvents. ${ }^{4,33,34}$ If the PEO-rich phase formed by the phase separation lies in a region of liquid-solid separation, crystallization occurs only in this phase. Thus, we infer that in PEO/DMAc, a liquid-liquid phase separation has occurred prior to or during the crystallization, and that the observed contour is a trace of phase boundary. According to this proposition, the crystallization occurs from a solution phase where the PEO concentration is greater than that of the initial solution. We note here that $A^{\prime}(\mathrm{TP})>A^{\prime}(\mathrm{DMAc})$ (see Table III), i.e., the energy barrier of the primary nucleation is greater for PEO/TP than for PEO/DMAc. This might imply that the phase separation promotes the primary nucleation for crystallization. Our microscopic observation in the early stage revealed that for PEO/DMAc, the particles as small as $50 \mu \mathrm{m}$ in diameter had already given birefringence, but we also observed areas without ap- 
parent birefringence inside the contour of the particle where PEO seems to exist as an amorphous state. The latter observation suggests the occurrence of phase separation without crystallization. Thus, the crystallites shown in Figure 3 may have originated from droplets formed initially by the phase separation.

Based on the above discussion, we propose the following scenario for the crystallization in the PEO/DMAc system: 1) liquid-liquid phase separation first takes place, and this phase separation results in the formation of spherical droplets (domains) of PEO-rich phase, 2) primary nucleation occurs exclusively within the PEO-rich phase, and this is followed by crystal growth, and 3) ragged aggregates of axialites grow inside the domain accompanied by distortion of its spherical contour, and at the same time, the domain itself grows slowly as the crystallization proceeds. During the above slow growth of the domain, PEO is deposited on the domain surface from a more dilute PEO solution phase, and this leads to a smaller absolute value of $G$ than those in the PEO/TP system as we discussed earlier.

\section{CONCLUSIONS}

Present study on crystallization rates of PEO from three solvents revealed that the ratio of apparent primary nucleation rate to radial growth rate is highest for PEO/DMAc and lowest for PEO/TP. The observed size and number density of the crystallites differ in accordance with this finding. Most likely the observed radial growth from DMAc and toluene solutions occurs due to PEO deposition from a solution phase of less PEO content than that in the case of PEO/TP. From PEO/DMAc solutions, small crystallites composed of aggregated multi-layered lamellae with branching grew, and a contour outside each of the crystallites was also observed, the latter suggesting that liquid-liquid phase separation had occurred. Similar morphology to that of PEO/DMAc was observed for $\mathrm{PEO} /$ toluene, but no contour was found. The $\mathrm{PEO} / \mathrm{TP}$ system gives relatively large crystallites, and their architecture depends on supercooling in the temperature range used in our experiments. This dependence on supercooling seems to be mainly due to a difference in the frequency of branching of the lamella layers. No direct evidence of the occurrence of liquid-liquid phase separation for PEO/DMAc was obtained in this study. It was not possible to determine whether particles of less than say $10 \mu \mathrm{m}$ in diameter at an earlier stage were droplets formed by the liquid-liquid phase separation or pure crystalline nuclei. We are now making an effort to check the presence of the binodal line in the tempera- ture range of crystallization for the PEO/DMAc system.

Acknowledgment. We thank Kyoji Tsubakiyama for his help in preparation of samples with a vacuum line apparatus. This work was supported by a Grantin-Aid for Scientific Research (No. 10750647) from the Ministry of Education, Culture, Sports, Science and Technology of Japan.

\section{REFERENCES}

1. L. Mandelkern, J. Appl. Phys., 26, 443 (1955).

2. J. Boon and J. M. Azcue, J. Polym. Sci., A-2, 6, 885 (1968).

3. I. C. Sanchez and E. A. DiMarzio, Macromolecules, 4, 677 (1971).

4. A. Nakajima, F. Hamada, S. Hayashi, and T. Sumida, Kolloid Z. Z. Polym., 222, 10 (1968).

5. A. Nakajima, S. Hayashi, T. Korenaga, and T. Sumida, Kolloid Z. Z. Polym., 222, 124 (1968).

6. H. Kusuyama, N. Miyamoto, Y. Chatani, and H. Tadokoro, Polym. Commun., 24, 119 (1983).

7. a) J.-J. Point and C. Coutelier, J. Polym. Sci., Polym. Phys. Ed., 23, 231 (1985).

b) J.-J. Point, C. Coutelier, and D. Villers, J. Phys. Chem., 90, 3273 (1986).

8. E. D. T. Atkins, M. J. Hill, D. A. Jarvis, A. Keller, E. Sarhene, and J. S. Shapiro, Colloid Polym. Sci., 262, 22 (1984).

9. J.-M. Guenet and G. B. McKenna, Macromolecules, 21, 1752 (1988).

10. M. Klein and J.-M. Guenet, Macromolecules, 22, 3716 (1989).

11. M. Klein, A. Mathis, A. Menelle, and J.-M. Guenet, Macromolecules, 23, 4591 (1990).

12. N. Inaba, K. Sato, S. Suzuki, and T. Hashimoto, Macromolecules, 19, 1690 (1986).

13. N. Inaba, T. Yamada, S. Suzuki, and T. Hashimoto, Macromolecules, 21, 407 (1988).

14. T. Sasaki, Y. Yamamoto, and T. Takahashi, Polym. J., 30, 868 (1998).

15. T. Sasaki, Y. Yamamoto, and T. Takahashi, Polym. J., 32, 263 (2000).

16. M. Hashimoto, T. Amasaki, and T. Itoh, Kobunshi Ronbunshu, 56, 821 (1999).

17. M. Imai, K. Kaji, T. Kanaya, and Y. Sakai, Phys. Rev. B: Condens. Matter Mater. Phys., 52, 12696 (1995).

18. N. J. Terrill, P. A. Fairclough, E. Towns-Andrews, B. U. Komanschek, R. J. Young, and A. J. Ryan, Polymer, 39, 2381 (1998).

19. D. Turnbull and J. C. Fisher, J. Chem. Phys., 17, 71 (1949).

20. K. Amistead and G. Goldbeck-Wood, Adv. Polym. Sci., 100, 219 (1992).

21. J. D. Hoffman and R. L. Miller, Polymer, 38, 3151 (1997).

22. H. A. Kramers, Physica, 7, 284 (1940).

23. T. Sasaki, M. Kurita, T. Yabu, and T. Takahashi, Macromolecules, 28, 8528 (1995).

24. Pracella, Eur. Polym. J., 21, 551 (1985).

25. a) N. Ding and E. J. Amis, Macromolecules, 24, 3906 (1991). b) N. Ding and E. J. Amis, Macromolecules, 24, 6464 (1991). 
26. B. Lotz and A. J. Kovacs, Kolloid Z. Z. Polym., 209, 97 (1966).

27. B. Lotz, A. J. Kovacs, G. A. Bassett, and A. Keller, Kolloid Z. Z. Polym., 209, 115 (1966).

28. A. Keller and N. O’Connor, Polymer, 1, 163 (1960).

29. D. J. Blundell and A. Keller, J. Polym. Sci. B, 6, 433 (1968).

30. A. Keller and E. Pedemonte, J. Cryst. Growth, 18, 111 (1973).

31. H. D. Keith and F. J. Padden, J. Appl. Phys., 34, 2409 (1963).
32. S. Z. D. Cheng, J. Chen, J. S. Barley, A. Zhang, A. Habenschuss, and P. A. Guisti, Polymer, 31, 845 (1990).

33. A. Nakajima, H. Fujiwara, and F. Hamada, J. Polym. Sci. A-2, 4, 507 (1966).

34. P. J. Flory, in "Principles of Polymer Chemistry", Cornell University Press, Ithaca, N.Y., 1967. 